\title{
Solving Engineering, Project, and Production Management Problems through Modeling
}

\author{
Chien-Ho Ko \\ Editor-in-Chief
}

Editorial

Available online January 15, 2016

This issue presents five papers covering engineering management, project management, and production management. While distinct, these three fields frequently overlap and share common managerial concepts, e.g. solving problems through modeling.

"Impact Analysis of Headway Distributions on Single-Lane Roundabout Entry Capacity" by Liang Ren and Xiaobo Qu from Australia's Griffith University was awarded Best Paper Award at the 2015 (6th) International Conference on Engineering, Project, and Production Management, held in Gold Coast, Australia. Here, the paper has been extended to provide additional detail about entry capacity estimation for single-lane roundabouts. The authors present a capacity model which provides improved accuracy over current models.

In "Entrepreneurial Orientation vs. Innovativeness of Small and Medium Size Enterprises,” Ejdys examines the impact of entrepreneurial orientation on innovation in small and medium size enterprises. Three hypotheses are examined by Structural Equation Modeling, with results showing that proactiveness has a significant positive influence on innovativeness, and that risk taking is an intermediate variable for innovation mediated by proactivity.

"Recent Trends in Theory Use and Application within the Project Management Discipline” by Johnson et al. reviews over 270 project management research articles from 1999 to 2013 to identify the five most frequently cited theories in dealing with project management issues: stakeholder theory, fuzzy sets theory, utility theory, theory of constraints, and actor-network theory. These theories are frequently used as a foundation for model development (Butler et al., 2001; Pollack et al., 2013; Rand, 2000; Shan et al., 2011).

In "Are Stock Markets Driven More by Sentiments than Efficiency?," Lasek and Lasek investigate the relationship between online financial news and stock market performance. A regression model of stock returns against sentiment scores is used to find that human sentiment influences capital markets. Sentiment analysis could be taken as a quantitative approach to measure opinion with possible applications for product management.

Manufacturing system flexibility is a key concern for on-time production delivery (Karim et al., 2010). In
"Impact of Flexibility of Manufacturing System Components on Competitiveness of SMEs in Northern India,” Kaur et al., use a Structural Equation Model to analyze the impact of flexibility on small and mediumsized enterprises.

While these five papers come from different fields and focus on different subjects, they all feature the use of modeling techniques for problem solving. Some examine model use in problem domains while others develop new models to derive meaningful results from data. We hope these works will inspire readers to adapt existing or build new models for problem solving in engineering, project, and production management.

\section{References}

Butler, J., Morrice, D. J., and Mullarkey, P. W. (2001). A multiple attribute utility theory approach to ranking and selection. Management Science, 47(6), 800-816.

Karim, M.A., Samaranayake, P., Smith, A.J.R., Halgamuge, S.K. (2010). An on-time delivery improvement model for manufacturing organisations. International Journal of Production Research, 48(8), 2373-2394.

Pollack, J., Costello, K., Sankaran, S. (2013). Applying Actor-Network Theory as a sensemaking framework for complex organisational change programs. International Journal of Project Management, 31(8), 1118-1128.

Rand, G. K. (2000). Critical Chain: The theory of constraints applied to project management. International Journal of Project Management, 18(3), 173-177.

Shan, X., Hou, W., Ye, X., and Wu, C. (2011). Decisionmaking criteria of PPP projects: Stakeholder theoretic perspective. World Academy of Science, Engineering and Technology, 77, 696-700. 